\title{
Therapeutic uses of scleral contact lenses for ocular surface disease: patient selection and special considerations
}

This article was published in the following Dove Press journal:

Clinical Optometry

\author{
Jennifer S Harthan' \\ Ellen Shorter ${ }^{2}$ \\ 'Illinois College of Optometry, \\ Chicago, IL, USA; ${ }^{2}$ University of Illinois \\ at Chicago, Chicago, IL, USA
}

\begin{abstract}
Scleral lenses are being prescribed for the management of corneal irregularity, uncomplicated refractive error, and ocular surface disease. There are many potential therapeutic uses of scleral lenses in addition to providing similar benefits as corneal gas permeable lenses. Scleral contact lenses are a valuable therapeutic tool for patients with ocular surface disease as these lenses protect the ocular surface, provide continuous corneal hydration while providing optimal visual correction, and are often used in conjunction with other therapies.
\end{abstract}

Keywords: scleral lenses, ocular surface disease, contact lenses

\section{Introduction}

The prescription of scleral lenses for the management of corneal irregularity, uncomplicated refractive error, and ocular surface disease is increasing. ${ }^{1-5}$ The large diameter of the medical device completely covers the cornea protecting it with a fluid reservoir creating an ideal ocular surface environment and making these lenses particularly useful for ocular surface disease. The Scleral Lenses in Current Ophthalmic Practice Evaluation (SCOPE) study group in 2015 reported that $16 \%$ of scleral lenses are currently being prescribed for ocular surface disease, $74 \%$ for corneal irregularity, and $10 \%$ for uncomplicated refractive error. ${ }^{6}$ More than $80 \%$ of scleral lens prescribers reported fitting their first lens after 2005 and $>54 \%$ after $2010 .{ }^{6}$ This increase may be due to innovations in technology, advancements in educational platforms, and increased availability of commercial lenses from laboratories. ${ }^{6}$ There are many therapeutic uses of scleral lenses in addition to providing similar benefits as a corneal gas permeable lens by masking irregular astigmatism and reducing higher order aberrations in patients with corneal irregularity. ${ }^{7}$ The precorneal fluid reservoir hydrates the cornea while mechanically protecting the ocular surface from the shearing forces of the lids and environment. ${ }^{3,4}$ This review focuses on one of the least used indications for scleral lenses, ocular surface disease.

Scleral lenses differ from corneal gas permeable lenses based on lens diameter and fitting characteristics. Corneal gas permeable contact lenses are supported exclusively by the cornea and do not extend past the limbus. Scleral lenses are large-diameter gas permeable contact lenses that are supported by a tear reservoir, rest on the conjunctival tissue overlying the sclera, and vault the cornea and limbus. ${ }^{8}$ These lenses are unique when compared to corneal gas permeable contact lenses in that they maintain a fluid reservoir between the back surface of the contact lens and
Correspondence: Jennifer S Harthan Michigan Avenue, Chicago, IL 60616, USA Tel + I 3I2949 7I37

Email Jharthan@ico.edu 
the front surface of the eye. The Scleral Lens Education Society classifies scleral contact lenses based on diameter: semi-scleral (12.5-15 mm), mini-scleral (15-18 mm), and large scleral (18-25 mm). To simplify this, scleral contact lenses are defined as large-diameter rigid gas permeable lenses that rest entirely on the sclera ranging from $14 \mathrm{~mm}$ to $>20 \mathrm{~mm}$ in diameter. ${ }^{8-10}$

Scleral lenses were initially made out of glass until polymethyl methacrylate was available in the 1940s. Due to the low oxygen permeability (Dk) of the materials used, signs of corneal hypoxia were common. ${ }^{1,11}$ Rigid gas permeable scleral contact lenses were not readily available until the 1980s when Ezekiel demonstrated improved comfort and performance with higher oxygen permeability and reduced corneal hypoxia. ${ }^{12}$ In addition to the use of the materials with higher Dk, the risks of hypoxia can also be reduced by minimizing lens thickness and tear reservoir. ${ }^{13}$ Visser et al described the availability of toric scleral lenses in 2006 enabling more precise fitting and improved patient comfort. ${ }^{2,14}$

Scleral lenses may be designed with spherical, frontsurface toric, back-surface toric, and bitoric platforms to optimize both scleral alignment and visual acuity. ${ }^{2,14}$ Multifocal scleral lens designs are also available to improve near visual acuity for presbyopic patients. When fitting scleral lenses for ocular surface disease, materials with high Dk values are recommended to minimize corneal hypoxia. ${ }^{15,16}$ Advancements in materials, lens designs, fitting techniques, and industry innovations have led to improved performance, increasing the indications for which they are being prescribed by eye care practitioners.

\section{Patient selection}

There are many patients with ocular surface disease who could potentially benefit from scleral lenses. The Tear Film and Ocular Surface Society (TFOS) Dry Eye Workshop II (DEWS II) report defined and classified patients based on their symptoms and signs of ocular surface disease. ${ }^{17}$ Individuals who are symptomatic with signs of ocular surface disease as well as those with neurotrophic conditions who have signs of ocular surface disease without symptoms are excellent candidates. These individuals will show clinical improvement in corneal punctate staining and filamentary keratitis with scleral lens use. A third group that may benefit from scleral lens therapy are individuals with keratoneuralgia or neuropathic pain who have symptoms of dry eye without signs of ocular surface disease. ${ }^{17,18}$ Neuropathic pain is often chronic due to damage or disease affecting the somatosensory nervous system, and neurosensory dysfunction may account for the lack of association between signs and symptoms in some patients. ${ }^{19}$ It has been suggested that ocular surface pain that develops following corneal nerve disruption represents a hypersensitivity of the ocular somatosensory nerves. ${ }^{20}$ In addition, patients may experience increased or exaggerated conjunctival and scleral pain due to secondary hyperalgesia. $^{21,22}$ Scleral lenses may help mitigate symptoms and disrupt the pain cycle in some of these patients even though they present with a "normal" appearing ocular surface.

While there are many potential benefits of scleral lenses for patients with ocular surface disease, they are not generally recommended as the initial therapy. In the past, therapeutic soft lenses have been recommended as a primary therapeutic lens option for some ocular surface conditions due to well-described fitting processes of soft contact lenses, the wide availability of the lenses, and well-documented results in the literature. ${ }^{23}$ There are also therapeutic soft lenses that are approved by US Food and Drug Administration for overnight or extended wear. These lenses are inexpensive and readily available, and multiple reports have shown the clinical benefits of them for patients with chronic ocular graft-versus-host disease (GVHD). ${ }^{24-26}$ However, for patients with significant ocular surface disease such as nonhealing epithelial defects, scleral lenses have proven to be beneficial in retaining a fluid-ventilated design allowing oxygenated precorneal fluid reservoir providing continuous corneal hydration with minimal corneal contact. ${ }^{27,28}$ Patients who are diabetic, are immunocompromised, or have nonhealing epithelial defects should be closely monitored for potential complications related to scleral lens use. Concomitant prophylactic antibiotic use can be considered in patients using therapeutic lenses; however, their use remains controversial. ${ }^{29}$

The overall goal when fitting patients with scleral lenses for ocular surface disease is to mitigate symptoms, and several case series have demonstrated that patients experience relief from or resolution of dryness, pain, irritation, and photophobia when wearing scleral lenses..$^{2,3,28}$ The 2017 TFOS DEWS II report recommended therapeutic contact lenses (soft bandage contact lenses and rigid scleral lenses) in step 3 of the management and treatment recommendations for dry eye disease. ${ }^{18}$ A 2015 SCOPE survey of scleral lens prescribers reported similar findings; scleral lenses were reported as being prescribed by practitioners after topical lubricants, topical steroids, cyclosporine, and punctal occlusion. ${ }^{5}$ For challenging medical conditions, scleral lenses can be used in combination with other therapies (topical lubricants, 
cyclosporine, topical corticosteroids, punctal occlusion, topical autologous serum, amniotic membrane grafting, and tarsorrhaphy) to delay or prevent surgery. ${ }^{30-33}$ Scleral lenses are a valuable therapeutic tool for patients with ocular surface disease as they protect the ocular surface, provide continuous corneal hydration while providing optimal visual correction, and can be used in conjunction with other therapies. ${ }^{2,3,27,28,34}$

There are no age restrictions when fitting scleral lenses. Pediatric patients may be fit, and parents or caregivers may be trained to assist with safe device application and removal. Geriatric patients should not be excluded due to advanced age. A study of patients fit with Prosthetic Replacement of the Ocular Surface Ecosystem (PROSE; BostonSight, Needham, MA, USA) devices found no difference in time required for application and removal of devices between young and older age-groups. ${ }^{35}$ All patients could apply devices in $<5$ minutes by week 5 and remove devices in $<5$ minutes by week $3 .{ }^{35}$ There are also a number of tools such as light-emitting diode light sources and device stands that can be used to assist patients with safe application and removal if there are issues with poor best-corrected visual acuity or hand dexterity.

\section{Initial lens selection and evaluation}

When fitting scleral lenses for patients with ocular surface disease, it is first essential to select a lens that is large enough to completely clear and vault the cornea and limbal area. The diameter of the lens is often dependent on the surface area of the ocular surface that needs rehabilitation. It may be beneficial to fit a large overall diameter to provide a greater area of protection from surface dryness and desiccation. Patients with a partial tarsorrhaphy or areas of symblepharon may necessitate a smaller diameter scleral lens. Once an initial lens is fit on the patient's eye, the practitioner must ensure that there is no central corneal touch or application bubbles.

The amount of clearance between the central cornea and the lens should be evaluated using an optic section at the slit lamp or with optical coherence tomography imaging. At the slit lamp, clearance can be estimated by comparing the center thickness of lens to the thickness of the post-tear film reservoir. Fluorescein dye can be placed in the bowl of the lens, and white light optic section can be used to help identify areas of corneal or limbal touch. If there is excessive central vaulting, a diagnostic lens with lesser sagittal depth or a flatter base curve should be tried, and if there is central touch, a diagnostic lens with higher sagittal depth or steeper base curve should be selected.

Several studies have looked at lens settling over time and have reported that, for most lens designs, there is likely a loss of central clearance of $\sim 80-100$ microns over the course of 1-8 hours of lens wear, usually plateauing around 2 hours of wear. ${ }^{36-38}$ It has also been reported that the viscosity of the solution used to fill the bowl of the lens does not influence the amount of lens settling on the eye. ${ }^{38}$

A study by Otchere et al evaluating scleral lenses of varying sagittal depth reported clearance loss after 1 hour of lens wear using the Visante optical coherence tomographer. ${ }^{37}$ The amount of settling has been reported to vary depending on wear time and lens design varying from $33.8 \pm 48.4$ microns after 1 hour, ${ }^{37}$ a decrease in 83 microns, ${ }^{39}$ and 113.7, 133.7, and 88.1 microns depending on design and diameter after 8 hours. ${ }^{40}$ Another study reported no difference in lens settling in 18-mm scleral lenses filled with nonpreserved saline versus more viscous nonpreserved gel and found 36.7 microns of central settling after 30 minutes and approximately double this amount, 70.0 microns, after 6 hours. ${ }^{38}$ There was no statistically significant change in subjective overrefraction after 6-8 hours of wear. ${ }^{39}$ Scleral lenses should be fit with minimal but complete corneal clearance. Giasson et al used 18-mm scleral lenses with 400 micron versus 200 micron clearance to demonstrate an in vivo $30 \%$ reduction in oxygen tension available to the cornea from $\mathrm{pO}_{2}$ of $9.07 \%$ to $6.19 \%{ }^{13}$

Once the central clearance is deemed appropriate, ensure that there is sufficient limbal clearance of $360^{\circ}$. The lens edge or haptic should also be modified to align with the sclera in each quadrant. If there are areas of excessive edge lift, the lens diameter can be decreased or a toric peripheral landing area can be used. Similarly, if the lens is too tight or vascular compression is observed, it can be loosened. Many patients will achieve superior scleral alignment by using a toric peripheral system that often has four quadrants. Patients with ocular surface disease often benefit from a large-diameter scleral lens, requiring toric haptics as the sclera has more toricity (Figure 1). ${ }^{2,8,14}$

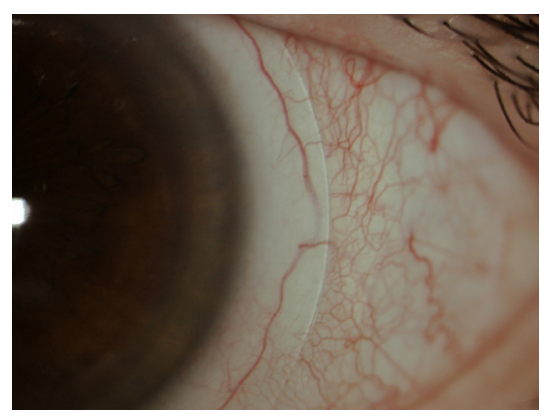

Figure I Scleral lens with nasal vascular compression that would benefit from toric peripheral system. 


\section{Safe handling}

Scleral devices require daily application, removal, and disinfection. It is important to instruct patients on proper application and removal with DMV ${ }^{\circledR}$ (DMV Corporation, Zanesville, OH, USA) scleral cups. Patients are instructed to fill their devices with preservative-free saline solution to prevent potential medicamentosa or toxicity to the ocular surface related to preservatives. There are a number of single-use preservative-free saline solutions as well as larger 4-oz bottles of preservative-free saline available. ScleralFil (Bausch + Lomb, Bridgewater, NJ, USA) is a unit-dose, buffered, nonpreserved saline solution indicated for rinsing and filling scleral lenses. LacriPure (Menicon Co. Ltd., Kasugai, Japan) is a unit-dose, nonbuffered, nonpreserved saline solution also indicated for rinsing and filling scleral lenses. Sodium chloride inhalation solution is an off-label, unit-dose, preservative-free solution, and PuriLens Plus saline (The LifeStyle Company, Inc., Freehold, NJ, USA) is a sterile, preservative-free, buffered saline solution; both solutions are used for filling and rinsing scleral lenses. Patients with severe ocular surface disease and those who are very sensitive may have a toxic response to buffer agents, and in most cases, a nonpreserved, nonbuffered solution is considered optimal.

After lens removal, a daily cleaner or multipurpose gas permeable solution can be prescribed to clean and disinfect lenses. Although manufacturer's directions and $38 \%$ of scleral lens prescribers' report instructing patients to use tap water to rinse lenses ${ }^{41}$ consideration should be made to instruct patients to rinse their lenses with saline. Hydrogen peroxide disinfection systems or multipurpose gas permeable lens solutions should be prescribed for overnight storage. It is not uncommon for individuals with severe dry eye or incomplete lid closure to report cloudy vision and lens fogging. These patients may need to use artificial tears or remove and reapply their lenses during the day. Removing and reapplying lenses with fresh preservative-free saline may be enough for some, while others may need to use a daily cleaner before reapplication. Patients with ocular surface disease often have surface nonwetting or deposits and may also benefit from Menicon Progent (Menicon) biweekly cleaner or from surface coatings such as Hydra-Peg ${ }^{\text {TM }}$ (Tangible Science LLC, Menlo Park, CA, USA) to help improve wettability, decrease friction, reduce surface deposits, and increase patient comfort.

Patients with ocular surface disease can use preservativefree artificial tears directly over the lens as needed throughout the day. Topical prescription medications should be used before or after scleral lens use, and patients should be instructed to wait at least 10 minutes before applying their lenses after their use. In patients with ocular surface disease, special consideration should be given to eliminate all benzalkonium chloride-preserved artificial tears and medications, particularly for those on glaucoma medications, as there are numerous alternative therapies available.

Patients who wear scleral lenses on an extended use basis such as in cases of persistent epithelial defects (PEDs) require very close medical monitoring. In a retrospective review of seven patients with PEDs, the patients were successfully treated with a standardized regimen of 24-hour PROSE device wear with daily office visits to remove, disinfect, and replace the fluid reservoir with preservative-free saline and a fourthgeneration fluoroquinolone antibiotic until re-epithelialized. ${ }^{42}$

\section{Therapeutic indications}

\section{Sjögren's syndrome (SS)}

SS is a chronic, progressive multisystem autoimmune disease that causes lymphocytic infiltration of the exocrine glands. ${ }^{43}$ The most common presenting symptoms are dry eye and dry mouth as the disease affects the lacrimal and salivary glands. ${ }^{43,44}$ As the disease progresses to extraglandular manifestations, patients have a risk of developing non-Hodgkin's B-cell lymphoma. ${ }^{43}$ Patients with SS often have severe ocular surface disease requiring preservative-free artificial tears, punctal plugs, topical cyclosporine, and scleral lenses. ${ }^{29,44} 46$

\section{Exposure keratopathy}

Exposure keratopathy may result from eyelid malposition due to trauma, surgery, ectropion, entropion, Bell's palsy, proptosis, and systemic diseases (Graves' disease). Inadequate or incomplete closure of the lids can result in ocular surface desiccation and breakdown of the corneal epithelium. ${ }^{47}$ Patients with exposure keratopathy typically complain of foreign body sensation, epiphora, blurred vision, photophobia, and varying levels of ocular discomfort. Common clinical signs include lagophthalmos, decreased tear meniscus height, reduced tear breakup time, filamentary keratitis, punctate epithelial erosions, and epithelial defects. If the condition is left untreated, patients with exposure keratopathy may have permanent vision loss due to neovascularization, corneal scarring, and stromal thinning. In extreme cases, corneal ulceration and perforation may occur. ${ }^{47}$

Traditional therapies of exposure keratopathy include a combination of artificial tears, gels and ointments, punctal plugs, topical steroids and antibiotics, moisture goggles, lid taping, gold eyelid weights, soft therapeutic lenses, partial or complete tarsorrhaphies, amniotic membrane grafts, or BOTOX injections to the levator muscle. Scleral lenses 
offer an alternative to tarsorrhaphy that has poor cosmesis and often results in loss of binocularity. In cases of extreme lagophthalmos, soft therapeutic lenses may displace on the eye due to dehydration. ${ }^{48}$ Scleral lenses have been successful in the management of patients with exposure keratopathy as the lens creates a liquid bandage shell, protecting the cornea from further desiccation, increasing hydration, and promoting epithelial surface healing (Figure 2). ${ }^{48,49}$

\section{Neurotrophic keratopathy}

Neurotrophic keratopathy results from impaired corneal innervation due to damage to the trigeminal nerve. Decreased corneal sensitivity or complete corneal anesthesia can result in epithelial keratopathy. The most common causes of neurotrophic keratopathy are herpes simplex and herpes zoster infections. Trigeminal neuralgia surgery, acoustic neuromas, diabetes, and toxicity from the chronic use of topical

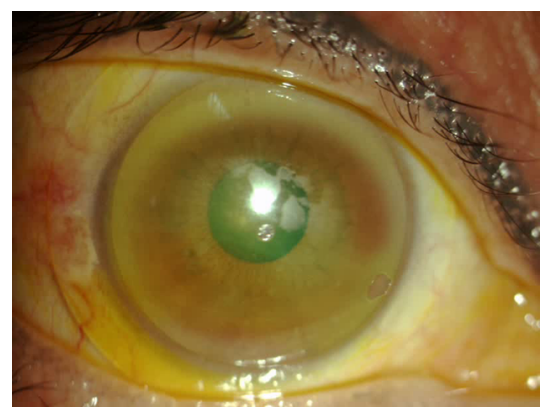

Figure 2 Patient with exposure keratopathy and poor lens surface wetting. Improvement was made with the addition of Hydra-Peg ${ }^{\mathrm{TM}}$ (Tangible Science LLC, Menlo Park, CA, USA). ocular medications may also result in corneal anesthesia. ${ }^{47,50}$ Anesthetic corneas are at risk for PEDs. Therapy options for neurotrophic corneas include preservative-free artificial tears and ointments, punctal plugs, partial tarsorrhaphy, topical antibiotics in cases of large epithelial defects, amniotic membranes, topical cyclosporine, oral doxycycline, therapeutic soft lenses, and scleral lenses. The protective liquid corneal bandage that the scleral lenses provide can heal the surface and prevent recurrence of the epithelial defect while preserving vision (Figure $3 \mathrm{~A}-\mathrm{C}$ ). ${ }^{47,50,51}$

\section{Limbal stem cell deficiency}

Limbal stem cell deficiency occurs due to congenital diseases such as ectodermal dysplasia or aniridia or can be acquired later in life. Inflammatory conditions such as StevensJohnson Syndrome or superior limbic keratoconjunctivitis, chemical or thermal injuries, chronic contact lens wear, and toxicity from topical medications may cause limbal stem cell deficiency ${ }^{52,53}$ Patients with limbal stem cell deficiency can present with complaints of decreased vision and light sensitivity and will have conjunctival epithelium growing onto the cornea with neovascularization and corneal scarring. ${ }^{52}$ The first step in the management of these patients is to eliminate all chemical preservative exposure and soft contact lens use. If there is no clinical improvement, antiinflammatory therapies should be considered and surgical limbal stem cell transplantation for more severe cases. ${ }^{52,54}$ Scleral lenses that completely vault the limbus are also an
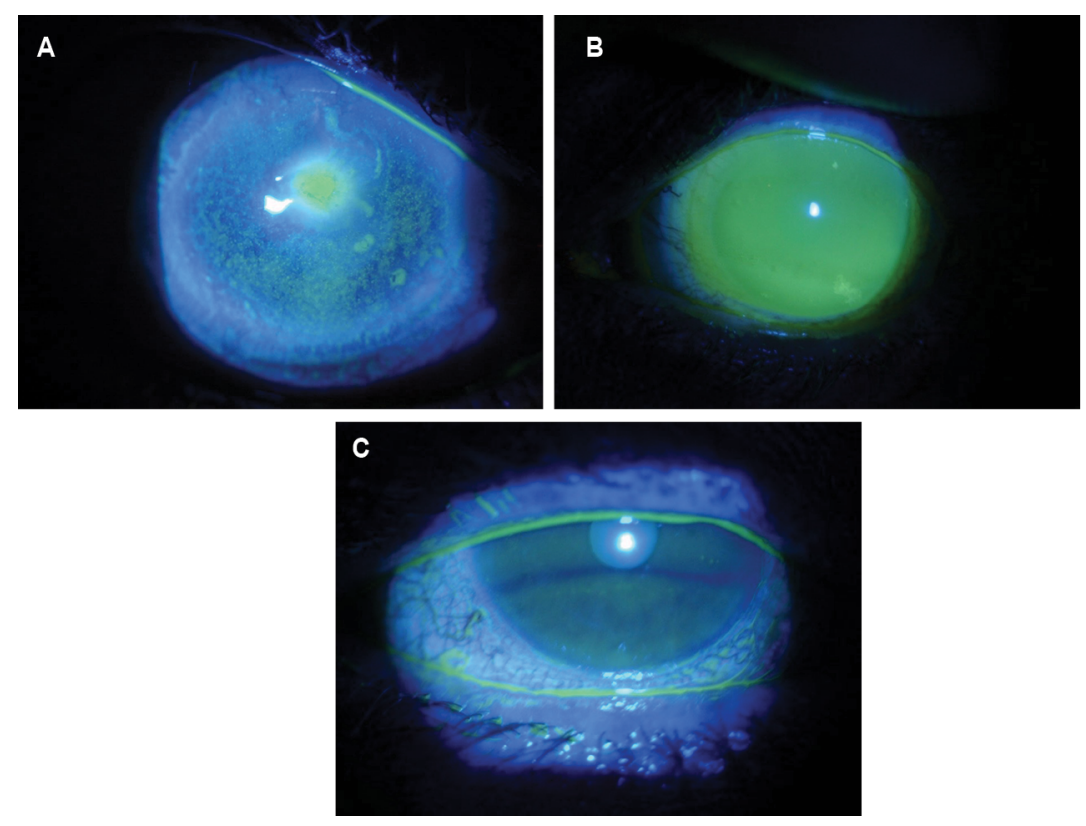

Figure 3 Patient with neurotrophic keratopathy whose nonhealing epithelial defect improved with therapeutic scleral lens use. Notes: (A) Non-healing epithelial defect; (B) scleral lens; (C) resolution of epithelial defect after I month of scleral lens therapy. 
excellent option for these patients. The lens can protect the limbus from mechanical trauma while maintaining a welllubricated ocular surface (Figure $4 \mathrm{~A}-\mathrm{C}$ ). ${ }^{53}$

\section{GVHD}

GVHD, an inflammatory disease that may be acute or chronic, is a complication following allogeneic stem cell transplantation for cancer. These individuals are at a risk of long-term immunosuppression and delayed healing. Keratoconjunctivitis sicca is a common sequela and is reported to affect $53 \%$ of individuals with chronic GVHD. ${ }^{55}$ These individuals may also have scleroderma-like findings including eyelid thickening, filamentary keratitis, and corneal ulceration. ${ }^{56}$ These patients present with complaints of ocular pain, foreign body sensation, blurred vision, photophobia, burning, and stinging associated with decreased quality of life and high morbidity. ${ }^{57-60}$ Therapeutic scleral lens therapy promotes healing of surface epitheliopathy while improving pain and photophobia associated with chronic ocular GVHD. ${ }^{61}$ Treatment is targeted to restore and maintain ocular surface homeostasis and reduce patients' symptoms and traditionally has included: topical lubricants, topical steroids or antibiotics, topical cyclosporine, punctal occlusion, autologous serum, and soft bandage contact lenses. Autologous serum tears have been shown to promote healing in patients with GVHD, ${ }^{62}$ and, although more studies are needed, they can be used to fill the device or diluted in the device with preservative-free solution. Scleral lenses can optimize vision, improve comfort, and protect the ocular surface in patients with GVHD (Figure 5A-C). ${ }^{63}$

\section{Post-penetrating keratoplasty (PK)}

While scleral lenses can play an important role in healing or maintaining the ocular surface in individuals with a previous PK, they must be closely monitored. The use of scleral lenses for individuals after PK can be controversial due to the unknown long-term effects of scleral lenses on the graft and endothelium. The fluid-filled device allows for healing of the corneal epithelium as well as masking of irregular corneal astigmatism improving best-corrected visual acuity. ${ }^{64,65}$ Patients should be very closely monitored for signs of corneal edema and acute corneal graft rejection. In addition to careful slit lamp examination, evaluating baseline and follow-up corneal pachymetry and specular microscopy may be considered. ${ }^{64,65}$ Normal endothelial cell density in a healthy adult patient is between 2000 and 2500 cells $/ \mathrm{mm}^{2}$. The endothelium begins to decompensate when the cell count drops $<500-1000$ cells $/ \mathrm{mm}^{2}$, leading to corneal edema, stromal haze, and reduced vision. ${ }^{66}$ If there are signs of corneal swelling or hypoxia, the patient may benefit from modification to the scleral lens fit including a change by decreasing
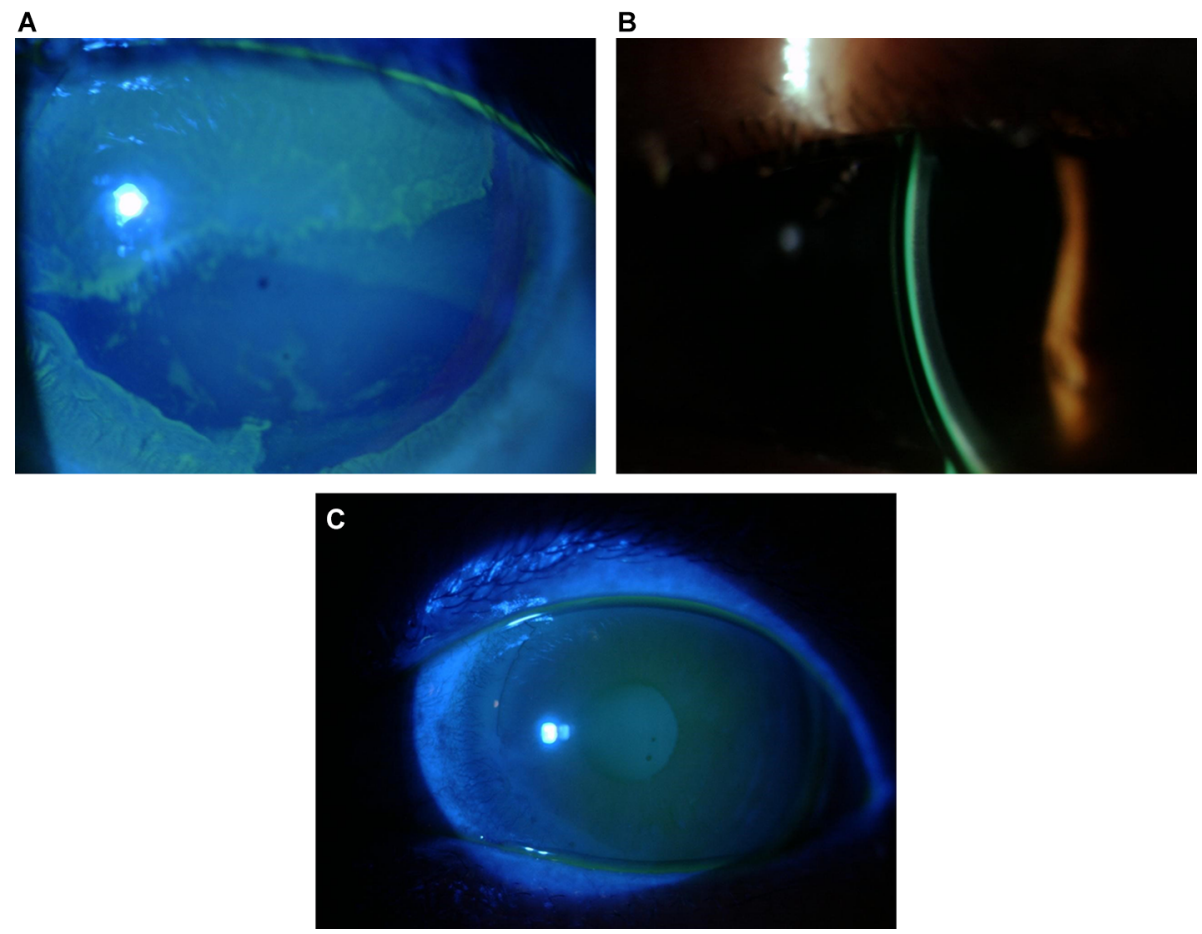

Figure 4 Patient with limbal stem cell disease managed with scleral lenses. Improvement was noted after 3 months of scleral lens wear Notes: (A) LSCD prescleral lens wear; (B) scleral lens fit; (C) improvement in LSCD post-scleral lens wear. Abbreviation: LSCD, limbal stem cell deficiency. 
A

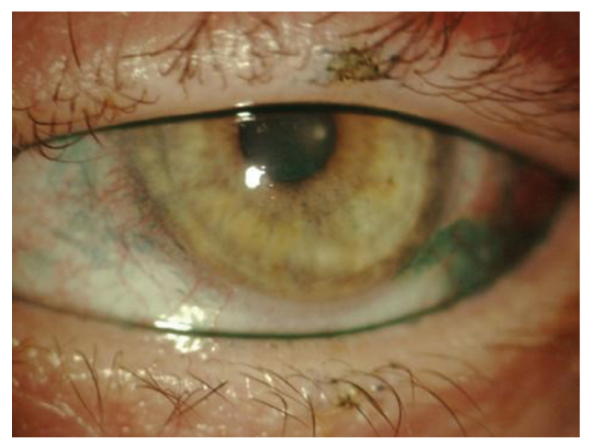

C
B

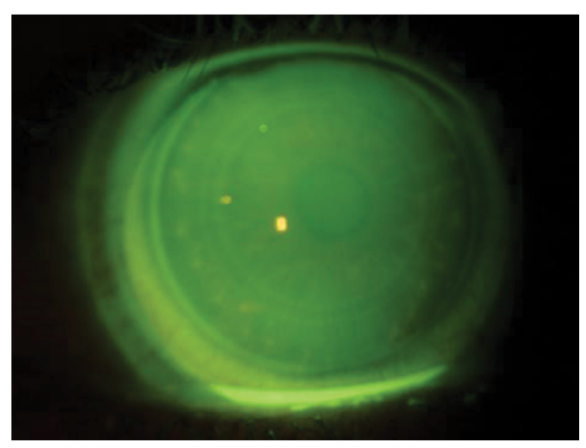

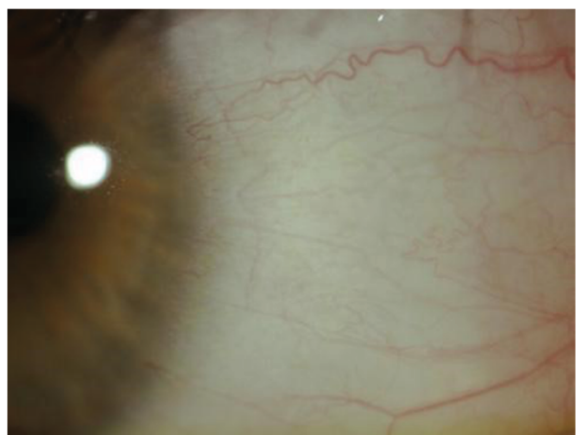

Figure 5 Patient with chronic graft-versus-host disease whose lissamine green staining improved following scleral lens therapy.

Notes: (A) Lissamine green staining; (B) scleral lens fit; (C) improvement in corneal staining following 3 months of daily scleral lens wear.

the sagittal profile, by flattening the peripheral haptic profile, by increasing the Dk of the material of the lens, by shortening patient wear schedule, or even by using fenestrations. ${ }^{14,64,65}$

\section{PEDs}

Patients with ocular surface disease and PEDs are difficult to manage as they are typically resistant to traditional therapies. PEDs occur when a damaged area of the cornea does not reepithelialize in the expected time frame and are associated with corneal dystrophies, neurotrophic keratitis, herpetic infections, Stevens-Johnson syndrome, ocular cicatricial pemphigoid, ocular trauma, or surgery. ${ }^{67}$ Patients may present with decreased vision and pain with episodes of recurrence taking months to resolve. PEDs must be treated aggressively as they can lead to corneal haze, irregular astigmatism, infectious keratitis, corneal melting, or perforation with loss of vision. ${ }^{14,34}$ Scleral lenses and PROSE devices have been shown to be beneficial in these refractory cases protecting the cornea from the shearing forces of eyelid blinking, maintaining a stable tear film, and promoting reepithelialization in patients with nonhealing epithelial defects. ${ }^{27,42,63}$

When prescribing any lens as an extended wear therapeutic option, the benefits must outweigh the risks. Several case reports have identified patients with PEDs who have successfully worn scleral lenses overnight without complications while promoting reepithelialization. ${ }^{42}$ There have been other cases of microbial keratitis in patients who have worn extended wear scleral lenses for PEDs. ${ }^{34}$ However, more recent case series using a prophylactic antibiotic have shown decreased risks associated with healing PEDs. There are reports on using two sets of scleral devices to heal PEDs. Guidelines for prescribing scleral devices in this manner include filling the device with preservative-free saline and preservative-free, fourth-generation fluoroquinolone antibiotics. Patients are instructed to remove, clean, and replace the solution every 12 hours during continuous wear with daily monitoring. ${ }^{23,27,34,68,69}$ Successful use of scleral lenses has been described in patients with PEDs as the postlens fluid chamber created by corneal vaulting is filled with nonpreserved saline to constantly lubricate the ocular surface, promoting healing of the epithelial defect. ${ }^{27,70-72}$

\section{Patient-reported poor vision or lens fogging}

Due to their underlying ocular surface disease, these patients are at an increased risk of poor lens wetting or lens fogging later in the day. Patients should be closely monitored for meibomian gland disease and treated aggressively. If patients complain of poor vision immediately after lens application, 
consideration should be given to current lens care recommendations. A separate alcohol-based lens cleaner can be prescribed in addition to an overnight hydrogen peroxide--based system. Individuals who use a lubricating ointment at bedtime may also benefit from an eye wash with a preservative-free saline prior to lens application in the morning. When patients complain of blurred vision or fogging later in the day, it is helpful to evaluate the patient later in the day after a few hours of continuous lens wear. At the slit lamp, evaluate whether there is lens surface nonwetting or if there is postlens tear film debris. Surface nonwetting can be improved with new surface coatings available or lens removal with cleaning and reapplication. Visser et al reported increased wear time and patient-reported comfort in toric scleral lenses compared with spherical scleral lenses. ${ }^{14}$ In our experience, toric peripheral systems can help limit debris formation, edge lift, and fogging. In addition, more viscous preservative-free filling solutions can be placed in the bowl of the lens to try to further delay fogging time.

\section{Discussion}

Individuals with signs of surface disease and symptoms who have failed traditional therapies are ideal candidates for scleral lens therapy. Therapeutic scleral lenses are also an option for individuals who are asymptomatic, but with signs such as neurotrophic keratopathy. While scleral lenses are often reserved as a later option when traditional therapies have failed, they are an important tool to heal the corneal epithelial and improve patient comfort as recommended in the 2017 TFOS DEWS II report.

The therapeutic effects of scleral lenses have been well documented. Individuals with keratoconjunctivitis sicca, limbal stem cell deficiency, neurotrophic keratitis, exposure keratitis, PEDs, and cicatrizing conjunctivitis can all benefit from therapeutic scleral lenses. Scleral lenses can play an important role as an adjunct to traditional therapy or as an alternative when traditional therapies fail. Scleral lenses may have advantages over traditional soft bandage lenses for the management of severe ocular surface disease recalcitrant to previous aggressive therapies. The unique design of a scleral lens allows the cornea to be completely vaulted while the lens rests entirely on the sclera. The postlens fluid reservoir filled with nonpreserved sterile saline constantly hydrates the cornea, facilitating the healing process of the ocular surface, preventing further desiccation of the ocular surface while simultaneously achieving optimal visual acuity. Scleral lenses also protect the ocular surface from keratinized or irregular lid margins and cicatricial entropion, increasing patient comfort. Management of patients with severe ocular surface disease often requires communication between health care providers as systemic conditions play a significant role in the healing process of the ocular surface. Patients who wear scleral lenses for ocular surface disease often require close follow-up and observation as compared to those who wear them for corneal irregularity or uncomplicated refractive error. The practitioner must understand the underlying disease process and determine whether the patient's symptoms at follow-up examination are due to the disease process or due to the lens fit.

Patients with ocular surface disease often benefit from a scleral lens with a larger overall diameter, which subsequently requires advanced toric peripheral systems in order to align with the sclera. When fitting scleral lenses in patients with ocular surface disease and especially those with PEDs, close medical monitoring is important to evaluate epithelial integrity and to ensure complete corneal and limbal vault. One model of fitting suggests erring on the side of fitting larger and looser versus a lens that is smaller in diameter and has a shallower sagittal depth. There are many options to enhance scleral lens wettability and decrease lens deposits. High-Dk materials are recommended when fitting these complex corneas, as well as detailed communication regarding safe wear and handling, as these patients often have multiple therapies that they are using. As scleral lenses create a postlens tear reservoir between the lens and the cornea, these lenses are thicker than corneal gas permeable lenses, potentially contributing to physiological edema at a subclinical level. ${ }^{13,16,73}$ Another model of fitting proposed by Michaud et al stated that the postlens tear layer should be no $>200$ microns in order to avoid corneal edema using a high-Dk $(>150)$ lens with a maximum central thickness of 250 microns. ${ }^{16}$ Regardless of preferred fitting methodology, careful observation is needed to monitor the long-term effects of scleral lenses on patients with ocular surface disease.

\section{Conclusion}

Scleral lenses are an effective therapeutic option in the management of ocular surface disease recalcitrant to traditional therapies. The overall goal when prescribing scleral lenses for ocular surface disease is to improve comfort while supporting the ocular surface and improving clinical signs of surface disease. Patients who are symptomatic with clinical signs and those who are asymptomatic but have clinical signs (neurotrophic keratopathy) are excellent candidates for longterm therapy with scleral lenses. 


\section{Disclosure}

Jennifer S Harthan - Allergan, Inc., Shire Pharmaceuticals, Metro, Tangible Science, and Ellen Shorter reports no conflicts of interest in this work.

\section{References}

1. Pullum KW, Whiting MA, Buckley RJ. Scleral contact lenses: the expanding role. Cornea. 2005;24:269-277.

2. Visser ES, Van der Linden BJ, Otten HM, Van der Lelij A, Visser R. Medical applications and outcomes of bitangential scleral lenses. Optom Vis Sci. 2013;90:1078-1085.

3. Visser ES, Visser R, van Lier HJ, Otten HM. Modern scleral lenses part I: clinical features. Eye Contact Lens. 2007;33:13-20.

4. Visser ES, Visser R, van Lier HJ, Otten HM. Modern scleral lenses part II: patient satisfaction. Eye Contact Lens. 2007;33:21-25.

5. Shorter E, Harthan J, Nau CB, et al. Scleral Lenses in the Management of Corneal Irregularity and Ocular Surface Disease. Eye Contact Lens. Epub 2017 Sep 29

6. Nau CB, Harthan J, Shorter E, et al. Demographic characteristics and prescribing patterns of scleral lens fitters: the SCOPE study. Eye Contact Lens. Epub 2017 Jun 14.

7. Marsack JD, Ravikumar A, Nguyen C, et al. Wavefront-guided scleral lens correction in keratoconus. Optom Vis Sci. 2014;91:1221-1230.

8. Van der Worp E. A Guide to Scleral Lens Fitting. 2nd ed. Forest Grove, OR: Pacific University; 2015.

9. DeNaeyer G, Sanders DR. sMap3D corneo-scleral topographer repeatability in scleral lens patients. Eye Contact Lens. Epub 2017 Jul 21.

10. DeNaeyer G, Sanders DR, Farajian TS. Surface coverage with single vs. multiple gaze surface topography to fit scleral lenses. Cont Lens Anterior Eye. 2017;40:162-169.

11. Pullum KW, Stapleton FJ. Scleral lens induced corneal swelling: what is the effect of varying Dk and lens thickness? CLAOJ. 1997;23:259-263.

12. Ezekiel D. Gas permeable haptic lenses. J Br Contact Lens Assoc 1983;6(4):158, 160-161.

13. Giasson CJ, Morency J, Melillo M, Michaud L. Oxygen tension beneath scleral lenses of different clearances. Optom Vis Sci. 2017;94: 466-475.

14. Visser ES, Visser R, Van Lier HJ. Advantages of toric scleral lenses. Optom Vis Sci. 2006;83:233-236.

15. Michaud L. For a better understanding of scleral contact lens-related pathophysiology. Invest Ophthalmol Vis Sci. 2014;55:6430.

16. Michaud L, van der Worp E, Brazeau D, Warde R, Giasson CJ. Predicting estimates of oxygen transmissibility for scleral lenses. Cont Lens Anterior Eye. 2012;35:266-271.

17. Craig JP, Nichols KK, Akpek EK, et al. TFOS DEWS II definition and classification report. Ocul Surf. 2017;15:276-283.

18. Jones L, Downie LE, Korb D, et al. TFOS DEWS II management and therapy report. Ocul Surf. 2017;15:575-628.

19. Belmonte C, Nichols JJ, Cox SM, et al. TFOS DEWS II pain and sensation report. Ocul Surf. 2017;15:404-437.

20. Levitt AE, Galor A, Weiss JS, et al. Chronic dry eye symptoms after LASIK: parallels and lessons to be learned from other persistent postoperative pain disorders. Mol Pain. 2015;11:21

21. Rosenthal P, Baran I, Jacobs DS. Corneal pain without stain: is it real? Ocul Surf. 2009;7:28-40.

22. Galor A, Zlotcavitch L, Walter SD, et al. Dry eye symptom severity and persistence are associated with symptoms of neuropathic pain. $\mathrm{Br}$ J Ophthalmol. 2015;99:665-668.

23. Pullum K, Buckley R. Therapeutic and ocular surface indications for scleral contact lenses. Ocul Surf. 2007;5:40-48.

24. Russo PA, Bouchard CS, Galasso JM. Extended-wear silicone hydrogel soft contact lenses in the management of moderate to severe dry eye signs and symptoms secondary to graft-versus-host disease. Eye Contact Lens. 2007;33:144-147.
25. Inamoto Y, Sun YC, Flowers ME, et al. Bandage soft contact lenses for ocular graft-versus-host disease. Biol Blood Marrow Transplant. 2015;21:2002-2007.

26. Stoyanova EI, Otten HM, Wisse R, Rothova A, Riemens A. Bandage and scleral contact lenses for ocular graft-versus-host disease after allogeneic haematopoietic stem cell transplantation. Acta Ophthalmol. 2015;93:e604

27. Rosenthal P, Cotter JM, Baum J. Treatment of persistent corneal epithelial defect with extended wear of a fluid-ventilated gas-permeable scleral contact lens. Am J Ophthalmol. 2000;130:33-41.

28. Rosenthal P, Croteau A. Fluid-ventilated, gas-permeable scleral contact lens is an effective option for managing severe ocular surface disease and many corneal disorders that would otherwise require penetrating keratoplasty. Eye Contact Lens. 2005;31:130-134.

29. Foulks GN, Forstot SL, Donshik PC, et al. Clinical guidelines for management of dry eye associated with Sjogren disease. Ocul Surf. 2015;13:118-132.

30. Donnenfeld ED, Selkin BA, Perry HD, et al. Controlled evaluation of a bandage contact lens and a topical nonsteroidal anti-inflammatory drug in treating traumatic corneal abrasions. Ophthalmology. 1995;102:979-984.

31. Panda A, Pushker N, Bageshwar LM. Lateral tarsorrhaphy: is it preferable to patching? Cornea. 1999;18:299-301.

32. Jeng BH. Use of autologous serum in the treatment of ocular surface disorders. Arch Ophthalmol. 2011;129:1610-1612.

33. Jeng BH, Dupps WJ, Jr. Autologous serum 50\% eyedrops in the treatment of persistent corneal epithelial defects. Cornea. 2009;28:1104-1108.

34. Lim P, Ridges R, Jacobs DS, Rosenthal P. Treatment of persistent corneal epithelial defect with overnight wear of a prosthetic device for the ocular surface. Am J Ophthalmol. 2013;156:1095-1101.

35. Kornberg DL, Dou E, Wang Y, et al. Clinical experience with PROSE fitting: significance of diagnosis and age. Eye Contact Lens. 2016;42:124-128.

36. Vincent SJ, Alonso-Caneiro D, Collins MJ. The temporal dynamics of miniscleral contact lenses: Central corneal clearance and centration. Cont Lens Anterior Eye. 2018:41(2):162-168.

37. Otchere H, Jones LW, Sorbara L. Effect of time on scleral lens settling and change in corneal clearance. Optom Vis Sci. 2017;94:908-913.

38. Courey C, Michaud L. Variation of clearance considering viscosity of the solution used in the reservoir and following scleral lens wear over time. Cont Lens Anterior Eye. 2017;40:260-266.

39. Bray C, Britton S, Yeung D, Haines L, Sorbara L. Change in overrefraction after scleral lens settling on average corneas. Ophthalmic Physiol Opt. 2017;37:467-472.

40. Kauffman MJ, Gilmartin CA, Bennett ES, Bassi CJ. A comparison of the short-term settling of three scleral lens designs. Optom Vis Sci. 2014;91:1462-1466.

41. Harthan J, Nau CB, Barr J, et al. Scleral lens prescription and management practices: the SCOPE study. Eye Contact Lens. Epub 2017 Apr 6.

42. Ciralsky JB, Chapman KO, Rosenblatt MI, et al. Treatment of refractory persistent corneal epithelial defects: a standardized approach using continuous wear PROSE therapy. Ocul Immunol Inflamm. 2015;23:219-224.

43. Vitali C, Bombardieri S, Jonsson R, et al. Classification criteria for Sjogren's syndrome: a revised version of the European criteria proposed by the American-European Consensus Group. Ann Rheum Dis. 2002;61:554-558.

44. Fernandes M, Sharma S. Polymicrobial and microsporidial keratitis in a patient using Boston scleral contact lens for Sjogren's syndrome and ocular cicatricial pemphigoid. Cont Lens Anterior Eye. 2013;36:95-97.

45. Lemp MA. Advances in understanding and managing dry eye disease. Am J Ophthalmol. 2008;146:350-356.

46. Foster CS, Sainz De La Maza M. Ocular cicatricial pemphigoid review. Curr Opin Allergy Clin Immunol. 2004;4:435-439.

47. Grey F, Carley F, Biswas S, Tromans C. Scleral contact lens management of bilateral exposure and neurotrophic keratopathy. Cont Lens Anterior Eye. 2012;35:288-291.

48. Williams ZR, Aquavella JV. Management of exposure keratopathy associated with severe craniofacial trauma. J Cataract Refract Surg. 2007;33:1647-1650. 
49. Harthan JS. Therapeutic use of mini-scleral lenses in a patient with Graves' ophthalmopathy. J Optom. 2014;7:62-66.

50. Nishida T, Yanai R. Advances in treatment for neurotrophic keratopathy. Curr Opin Ophthalmol. 2009;20:276-281.

51. Goins KM. New insights into the diagnosis and treatment of neurotrophic keratopathy. Ocul Surf. 2005;3:96-110.

52. Ghahari ESD, Holland EJ, Djalilian AR. Ocular Surface Disease. Management of Limbal Stem Cell Deficiency. Cham, Switzerland: Springer International Publishing; 2018:281-306.

53. Rossen J, Amram A, Milani B, et al. Contact lens-induced limbal stem cell deficiency. Ocul Surf. 2016;14:419-434.

54. Thomasen H, Steuhl KP, Meller D. [Rehabilitation following limbal stem cell deficiency: current treatment options and future developments]. Klin Monbl Augenheilkd. Epub 2017 Sep 12. German.

55. Allan EJ, Flowers ME, Lin MP, Bensinger RE, Martin PJ, Wu MC. Visual acuity and anterior segment findings in chronic graft-versus-host disease. Cornea. 2011;30:1392-1397.

56. Nassar A, Tabbara KF, Aljurf M. Ocular manifestations of graft-versushost disease. Saudi J Ophthalmol. 2013;27:215-222.

57. Kanda J, Brazauskas R, Hu ZH, et al. Graft-versus-host disease after hla-matched sibling bone marrow or peripheral blood stem cell transplantation: comparison of north american caucasian and japanese populations. Biol Blood Marrow Transplant. 2016;22:744-751.

58. Ferrara JL, Deeg HJ. Graft-versus-host disease. $N$ Engl J Med. 1991;324:667-674.

59. Ferrara JL, Levine JE, Reddy P, Holler E. Graft-versus-host disease. Lancet. 2009;373:1550-1561.

60. Westeneng AC, Hettinga Y, Lokhorst H, Verdonck L, van Dorp S, Rothova A. Ocular graft-versus-host disease after allogeneic stem cell transplantation. Cornea. 2010;29:758-763.

61. Schornack MM, Baratz KH, Patel SV, Maguire LJ. Jupiter scleral lenses in the management of chronic graft versus host disease. Eye Contact Lens. 2008;34:302-305.
62. Ogawa Y, Okamoto S, Mori T, et al. Autologous serum eye drops for the treatment of severe dry eye in patients with chronic graft-versus-host disease. Bone Marrow Transplant. 2003;31:579-583.

63. Chiu GB, Theophanous C, Irvine JA. PROSE treatment in atypical ocular graft-versus-host disease. Optom Vis Sci. 2016;93: 1444-1448.

64. Severinsky B, Behrman S, Frucht-Pery J, Solomon A. Scleral contact lenses for visual rehabilitation after penetrating keratoplasty: long term outcomes. Cont Lens Anterior Eye. 2014;37:196-202.

65. Barnett M, Lien V, Li JY, Durbin-Johnson B, Mannis MJ. Use of scleral lenses and miniscleral lenses after penetrating keratoplasty. Eye Contact Lens. 2016;42:185-189.

66. Okumura N, Kinoshita S, Koizumi N. Application of Rho kinase inhibitors for the treatment of corneal endothelial diseases. J Ophthalmol. 2017;2017:2646904.

67. Blackmore SJ. The use of contact lenses in the treatment of persistent epithelial defects. Cont Lens Anterior Eye. 2010;33:239-244.

68. Cotter JM, Rosenthal P. Scleral contact lenses. JAm Optom Assoc. 1998;69: $33-40$.

69. Tappin MJ, Pullum KW, Buckley RJ. Scleral contact lenses for overnight wear in the management of ocular surface disorders. Eye (Lond). 2001;15:168-172.

70. Schornack MM. Scleral lenses: a literature review. Eye Contact Lens. 2015;41:3-11.

71. Jacobs DS. Update on scleral lenses. Curr Opin Ophthalmol. 2008;19: 298-301.

72. Jacobs DS, Rosenthal P. Boston scleral lens prosthetic device for treatment of severe dry eye in chronic graft-versus-host disease. Cornea. 2007;26:1195-1199.

73. Walker MK, Bergmanson JP, Miller WL, Marsack JD, Johnson LA. Complications and fitting challenges associated with scleral contact lenses: a review. Cont Lens Anterior Eye. 2016;39:88-96.
Clinical Optometry

\section{Publish your work in this journal}

Clinical Optometry is an international, peer-reviewed, open access journa publishing original research, basic science, clinical and epidemiological studies, reviews and evaluations on clinical optometry. All aspects of patient care are addressed within the journal as well as the practice of optometry including economic and business analyses. Basic and clinical Submit your manuscript here: https://www.dovepress.com/clinical-optometry-journal

\section{Dovepress}

research papers are published that cover all aspects of optics, refraction and its application to the theory and practice of optometry. The manuscrip management system is completely online and includes a very quick and fair peer-review system, which is all easy to use. Visit http://www.dovepress. com/testimonials.php to read real quotes from published authors. 